\title{
Role of van der Waals corrections in first principles simulations of alkali metal ions in aqueous solutions
}

Takashi Ikeda, and Mauro Boero

Citation: The Journal of Chemical Physics 143, 194510 (2015); doi: 10.1063/1.4935932

View online: https://doi.org/10.1063/1.4935932

View Table of Contents: http://aip.scitation.org/toc/jcp/143/19

Published by the American Institute of Physics

\section{Articles you may be interested in}

Perspective: How good is DFT for water?

The Journal of Chemical Physics 144, 130901 (2016); 10.1063/1.4944633

Structure and dynamics of aqueous solutions from PBE-based first-principles molecular dynamics simulations The Journal of Chemical Physics 145, 154501 (2016); 10.1063/1.4964865

Probing the structural and dynamical properties of liquid water with models including non-local electron correlation

The Journal of Chemical Physics 143, 054506 (2015); 10.1063/1.4927325

Redox levels in aqueous solution: Effect of van der Waals interactions and hybrid functionals

The Journal of Chemical Physics 143, 244508 (2015); 10.1063/1.4938189

Hydration structure of salt solutions from ab initio molecular dynamics

The Journal of Chemical Physics 138, 014501 (2013); 10.1063/1.4772761

Hydration of alkali ions from first principles molecular dynamics revisited

The Journal of Chemical Physics 126, 034501 (2007); 10.1063/1.2424710

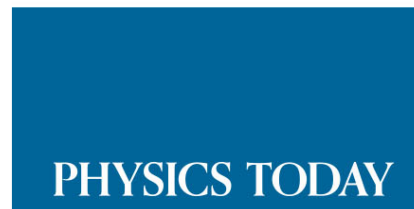




\title{
Role of van der Waals corrections in first principles simulations of alkali metal ions in aqueous solutions
}

\author{
Takashi lkeda ${ }^{1, a)}$ and Mauro Boero ${ }^{2}$ \\ ${ }^{1}$ Condensed Matter Science Division, Quantum Beam Science Center, Japan Atomic Energy Agency (JAEA), \\ 1-1-1 Kouto, Sayo-cho, Sayo-gun, Hyogo 679-5148, Japan \\ ${ }^{2}$ Institut de Physique et Chimie des Matériaux de Strasbourg, UMR 7504 CNRS and University of Strasbourg, \\ 23 Rue du Loess, 67034 Strasbourg, France
}

(Received 26 August 2015; accepted 5 November 2015; published online 19 November 2015)

\begin{abstract}
By resorting to a novel implementation of the first-principles-based van der Waals correction (vdWC) based on maximally localized Wannier functions (MLWFs), we inspect its performance and assess its reliability for aqueous solutions of alkali metal ions. In our implementation of vdWCs, an efficient extrapolation scheme is introduced to allow for affordable first principles molecular dynamics avoiding the explicit recalculation of MLWFs at each step. We find that vdWCs, when added to the widely used revPBE gradient corrected functional, influence substantially both structural and dynamical properties of water molecules, with particular emphasis on the hydration shell of the alkali cations. These effects are more evident for strong structure-making and -breaking cationic species. Moreover, self-diffusion coefficients and reorientation correlation times of solvating water molecules change systematically, showing a trend in better agreement with experiments with respect to simulations neglecting the long-range dispersion contributions. (C) 2015 AIP Publishing LLC. [http://dx.doi.org/10.1063/1.4935932]
\end{abstract}

\section{INTRODUCTION}

Due to the ubiquitous presence and crucial roles that hydrated alkali metal cations play in several fields ranging from solution chemistry to fuel cells, and biological systems, ${ }^{1-10}$ having a reliable computational setup for their simulation is nowadays of fundamental importance. Hydration is a crucial issue, because water is a universal solvent able to dissolve more substances than any other liquid medium and, in this respect, wherever water is present, either in an electrolytic device or in a living cell, it becomes a general vector for the transport of chemicals. It is then not surprising that special attention has so far been given to pure water, ${ }^{11-15}$ particularly in the field of first principles molecular dynamics (FPMD) simulations within the density functional theory ${ }^{16}$ (DFT) framework. In this context, several groups have recently brought to the general attention, the non-negligible role of van der Waals (vdW) interactions ${ }^{17-26}$ for a proper description of structure, hydrogen bonding, density, and pressure of liquid water. $^{27-38}$

Focusing, instead, on the solutes, alkali metal ions solvated in water play a prominent role on one hand because of their fundamental importance in living organisms and their interaction with the surrounding environment. For instance, $\mathrm{Na}^{+}$and $\mathrm{K}^{+}$share the same concentration $(460$ and $10 \mathrm{mM}$, respectively) both in sea water and in the extracellular environment of mammalian blood cell, whereas their respective concentrations are roughly reversed if one looks inside the cell membrane. ${ }^{39,40}$ On the other hand, they are key ingredients in a wealth of technological applications

a)Electronic mail: ikeda.takashi@jaea.go.jp from electrocatalysis ${ }^{41}$ to solar cells. ${ }^{42}$ One of the most fundamental phenomena of alkali cations in aquatic physicalchemistry is their structure making and breaking properties. ${ }^{1,9}$ In fact, alkali metal ions are known to enhance or weaken the structuring of the hydrogen-bond (H-bond) network formed by $\mathrm{H}_{2} \mathrm{O}$ molecules depending on the solvated ionic species and their concentrations. This, in turn, affects diffusion and reorientation of water molecules, although different opinions about the influence of the cations on the properties of water have been proposed over the years. ${ }^{4-45}$

All former FPMD simulations ${ }^{46-53}$ including ours ${ }^{54-57}$ have been of great help in clarifying major issues such as the structure of first solvation shell, the infrared spectra, waterexchange processes, and, to a certain extent, depending on the length of the simulations, the diffusion properties of water molecules and alkali metal ions if included. However, to date, it remains unclear whether or not the contribution of $\mathrm{vdW}$ dispersion forces is equally important in the solute-solvent interactions, particularly for the fundamental case of alkali cations. To fill this gap, we consider here the alkali metal ions $\mathrm{Li}^{+}, \mathrm{Na}^{+}, \mathrm{K}^{+}, \mathrm{Rb}^{+}$, and $\mathrm{Cs}^{+}$focusing on both structural properties of the solvation shell and dynamical properties in terms of self-diffusion coefficient and reorientation correlation time of water molecules. By analyzing the effects of $\mathrm{vdW}$ interactions, we provide a clear picture of their role according to the nature of each cation. This systematic analysis allows to rationalize and to suggest improvements for the simulation of hydrated alkali metal cations in FPMD approaches. Specifically, we show which alkali metal ions are most affected by including vdW interactions and provide a detailed comparison with experiments to support our conclusions. 
The remainder of the paper is organized as follows. In Sec. II, we describe the computational method including some technical details relevant to the present study. In Sec. III, the structural properties of the first solvation shell and dynamics of water molecules in the aqueous solutions are discussed in detail and the performance of our implemented $\mathrm{vdW}$ corrections is assessed critically. Finally, in Sec. IV, we draw our conclusions.

\section{COMPUTATIONAL METHODS}

In all the simulations presented in this work, we use the FPMD approach within the Car-Parrinello ${ }^{58,59}$ (CPMD) scheme. The computational protocol is analogous to the one we used in the former works. ${ }^{54,55}$ As far as the choice of the exchange and correlation (XC) functional is concerned, we adopt the modification of the Perdew-Burke-Ernzerhof ${ }^{60}$ (PBE) formula proposed by Zhang and Yang, ${ }^{61}$ which has been shown to improve systematically total and atomization energies. Moreover, this specific generalized gradient approximation (GGA), referred to as revPBE, allows for an efficient first-principles (FP) inclusion of vdW corrections (vdWCs) written as

$$
E_{\mathrm{vdW}}=-\sum_{n<l} f\left(r_{n l}\right) C_{6, n l} / r_{n l}^{6} .
$$

In this expression, following a scheme based on maximally localized Wannier functions ${ }^{62-64}$ (MLWFs) originally introduced in Refs. 20 and 65, $r_{n l}$ is the distance between the $n$th MLWF center of one fragment (or non-bonded subsystem) and the $l$ th MLWF center of another fragment. In this work, the whole system is partitioned into fragments on the basis of the connectivity of the system in a given configuration as described below. The sum of Eq. (1) is taken over all the MLWF centers of pairs of fragments (of course, excluding repetitions, since $r_{n l}=r_{l n}$ ). The damping function $f\left(r_{n l}\right)$ is introduced to cutoff the unphysical behavior and to avoid double counting of the interactions at short fragment separations, analogously to what has been used and discussed in Refs. 17 and 65. The electronic structure, computed within the selected DFT-GGA scheme, is used to compute MLWFs. These allow for an unbiased partitioning of the charge density and can be computed on the fly during FPMD with a modest computational effort. Then, MLWF centers

$$
x_{n}=-\frac{L}{2 \pi} \mathfrak{J} m \log \left\langle w_{n}|\exp (-i 2 \pi \cdot x / L)| w_{n}\right\rangle
$$

with similar expressions along the other two Cartesian directions, and their spreads are used to compute the $C_{6, n l}$ coefficients according to the procedure proposed by the group of Silvestrelli. ${ }^{20,22}$ Here, $L$ is the length of the simulation cell along the $x$ direction and $w_{n}$ is the computed $n$th MLWF. In this work, our vdW-corrected revPBE is denoted as revPBE+vdW.

The valence-core interaction is described by a normconserving Troullier-Martins ${ }^{66}$ pseudopotential (PP) for H, O, $\mathrm{Na}$, and $\mathrm{K}$, and Goedecker ${ }^{67,68} \mathrm{PP}$ for $\mathrm{Li}, \mathrm{Rb}$, and $\mathrm{Cs}$ generated by using the relativistic Dirac equation for a single atom in the construction of PP. The semicore states of $1 s$ for $\mathrm{Li}, 2 s$ and $2 p$ for $\mathrm{Na}, 3 s$ and $3 p$ for $\mathrm{K}, 4 s$ and $4 p$ for $\mathrm{Rb}$, and $5 s$ and $5 p$ for
Cs are treated as valence states. Valence orbitals are expanded in a plane-wave basis set with an energy cutoff of $80 \mathrm{Ry}$ and periodic boundary conditions are applied to the simulation cell. The sampling of the Brillouin zone is limited to the $\Gamma$ point and the numerical integration of the CPMD equations of motion is done with a time step of 4 a.u. (0.0968 fs) and a fictitious electron mass of 400 a.u. These values have already been carefully benchmarked and shown to be able to ensure a good adiabaticity of the CPMD trajectories. ${ }^{69-71}$

Concerning the aqueous solutions for the various alkali metal ions, we use simulation systems identical to the ones used in our former studies. ${ }^{54,55}$ Namely, a cation is solvated by $63 \mathrm{H}_{2} \mathrm{O}$ molecules in a cubic simulation cell of side $L=12.417 \AA$ on which periodic boundary conditions are applied. The excess charge of the systems is compensated by a uniform background charge ${ }^{72}$ instead of adding explicit counterions. After equilibrating the system for $\sim 2 \mathrm{ps}$ at $300 \mathrm{~K}$, the trajectories were collected for at least $150 \mathrm{ps}$ to accumulate statistics. All the simulations were done in an $N V T$ canonical ensemble at $T=300 \mathrm{~K}$ using a Nosé-Hoover chains thermostat ${ }^{73-76}$ to control the temperature.

As already mentioned, our vdWCs scheme requires a virtual partitioning of the system into non-bonded fragments. For this purpose bonded pairs of atoms were identified based on the covalent bond radius of each constituent of the system. The actual values used in this work are 0.32 for $\mathrm{H}, 0.73$ for $\mathrm{O}$, 1.23 for $\mathrm{Li}, 1.54$ for $\mathrm{Na}, 2.03$ for $\mathrm{K}, 2.16$ for $\mathrm{Rb}$, and $2.35 \AA$ for Cs. Two atoms are considered as bonded if their distance is smaller than 1.35 times the sum of their given covalent bond radii, where 1.35 is a tolerance factor selected to safely distinguish intramolecular covalent $\mathrm{OH}$ bonds of an $\mathrm{H}_{2} \mathrm{O}$ from $\mathrm{H}$-bonds formed with its neighboring $\mathrm{H}_{2} \mathrm{O}$ molecules. According to this criterion, one alkali metal ion, on which the MLWF centers corresponding to the semicore states are also located, and $\mathrm{H}_{2} \mathrm{O}$ molecules in the first solvation shell constitute a single fragment. Each $\mathrm{H}_{2} \mathrm{O}$ molecule remaining in the system is treated as a single fragment as long as any intermolecular pairs of $\mathrm{H}$ and $\mathrm{O}$ atoms are assigned as nonbonded. Accordingly, our vdWCs are free from the double counting of short range interactions between an alkali ion and neighboring $\mathrm{H}_{2} \mathrm{O}$ molecules as well as between bonded pairs of $\mathrm{H}_{2} \mathrm{O}$ molecules if such pairs are present.

In our scheme, vdWCs are evaluated as already mentioned in terms of the center and spread of MLWFs. We recall that each MLWF center is located near the atom sites for ionic compounds (and lone pairs) or in the middle of covalent bonds, since they are associated with the chemical bonds they represent. This feature allows for a considerable reduction of the computational cost; a set of $C_{6, n l}$ coefficients in Eq. (1) computed, e.g., at time $t$ can be reused at later times $t+\Delta t$ along the dynamics while each MLWF center is simply extrapolated according to the movement of a reference position of the center of atom or covalent bond associated with each MLWF. This avoids the explicit calculation of MLWFs at each MD step. This procedure is used until the deviation of the atom positions exceeds the selected tolerance. When this occurs, a recalculation of MLWFs via unitary transformation of the Kohn-Sham orbitals is performed. In our implementation, the maximum deviation of atom positions, 
with respect to those in the configuration for which MLWFs were previously computed, is used as a discriminating quantity for the tolerance. The actual value of the tolerance was set to 5.0 a.u. in this work. Consequently, the update of MLWFs and the resulting $C_{6, n l}$ coefficients can be skipped for several thousands (around 5000) of CPMD steps, thus leading to a remarkable decrease of the overhead actually spent for our vdWCs. More precisely, for the systems simulated here, only a small fraction amounting to about $1 \%$ of the total computational cost is required for calculating the vdW part. We remark that the update of the $C_{6, n l}$ coefficients inevitably leads to small fluctuations of the total energy $(\sim 0.3 \mathrm{eV}$ or less in magnitude for our aqueous systems), which is still a well conserved quantity whenever the same set of $C_{6, n l}$ coefficients is reused instead than recomputed.

\section{RESULTS AND DISCUSSION}

The computed radial distribution functions (RDFs) $g_{\mathrm{MO}}(r)$ and $g_{\mathrm{MH}}(r)$ for the cations $\mathrm{M}^{+}(\mathrm{M}=\mathrm{Li}, \mathrm{Na}, \mathrm{K}, \mathrm{Rb}$, and $\mathrm{Cs})$ solvated in ambient water (AW) are shown in Fig. 1. A first noticeable effect of the vdW contribution can be seen in the first peaks of these RDFs. Indeed, the intensity of all the first peak located at 2.0,2.4, 2.8, 3.0, and $3.2 \AA$ in $g_{\mathrm{LiO}}(r)$, $g_{\mathrm{NaO}}(r), g_{\mathrm{KO}}(r), g_{\mathrm{RbO}}(r)$, and $g_{\mathrm{CsO}}(r)$, respectively, is slightly reduced by adding vdWCs, compared to the corresponding RDFs obtained using the bare revPBE. The effect of vdWCs is, instead, less visible on the remaining peaks, which are
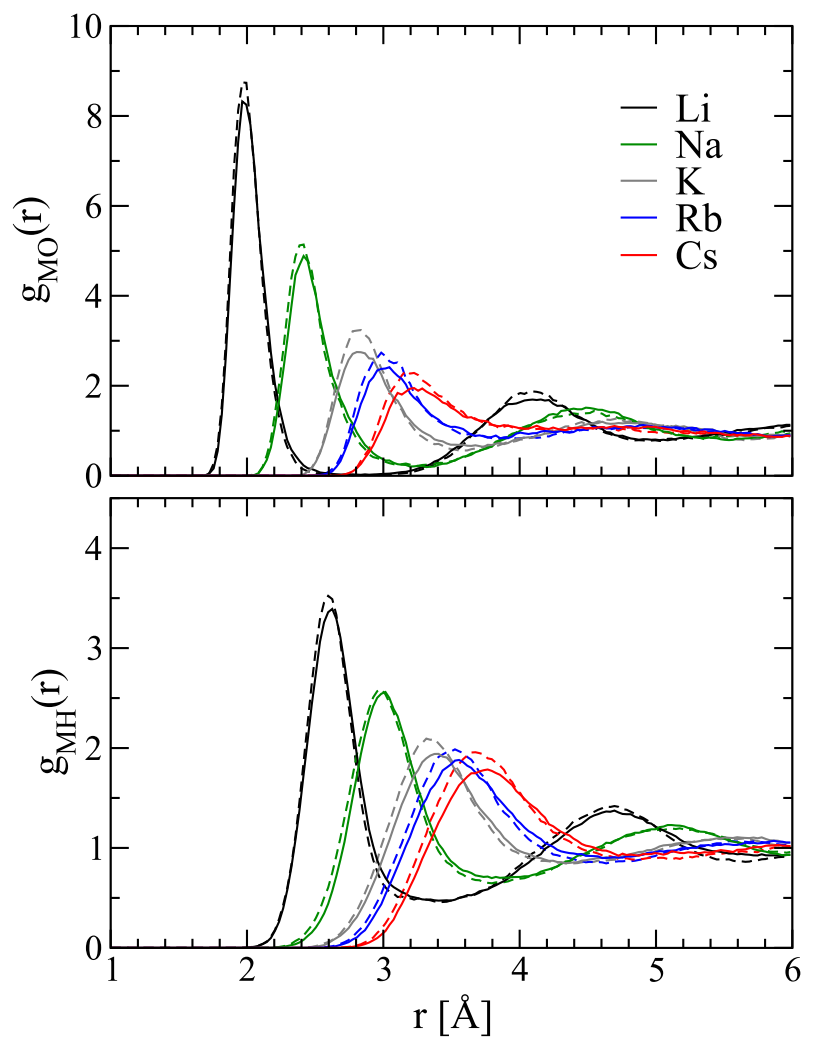

FIG. 1. RDFs $g_{\mathrm{MO}}(r)$ (upper) and $g_{\mathrm{MH}}(r)$ (lower) for alkali metal ion $\mathrm{M}^{+}$ $(\mathrm{M}=\mathrm{Li}, \mathrm{Na}, \mathrm{K}, \mathrm{Rb}$, and $\mathrm{Cs})$ in our liquid water obtained using revPBE (dashed) and revPBE+vdW (solid). nearly unaffected with the only exception of $\mathrm{Li}^{+}$, which still shows a slightly decreased intensity also in the second peak both in $g_{\mathrm{LiO}}(r)$ and in $g_{\mathrm{LiH}}(r)$. The positions of the first peak in $g_{\mathrm{LiO}}(r), g_{\mathrm{NaO}}(r), g_{\mathrm{KO}}(r)$, and $g_{\mathrm{CsO}}(r)$ are in good agreement with the available data for $\mathrm{M}^{+}-\mathrm{OH}_{2}$ distance obtained by $\mathrm{X}$ ray and neutron diffraction experiments performed on aqueous solutions of $\mathrm{Li}^{+}\left(1.95-2.28 \AA^{77,78}\right), \mathrm{Na}^{+}\left(2.39-2.42 \AA^{79-81}\right), \mathrm{K}^{+}$ (2.60-2.95 $\left.\AA^{79,82-84}\right)$, and $\mathrm{Cs}^{+}\left(2.95-3.21 \AA^{79,85}\right)$, respectively. The coordination number $(\mathrm{CN})$ of heavy alkali ions estimated using revPBE+vdW turns out to be 6.55 for $\mathrm{K}^{+}, 6.95$ for $\mathrm{Rb}^{+}$, and 7.07 for $\mathrm{Cs}^{+}$. These values are $0.21,0.44$, and 0.62 smaller than the respective estimates using revPBE. Conversely, the $\mathrm{CNs}$ of $\mathrm{Li}^{+}$(4.00) and $\mathrm{Na}^{+}$(5.13) computed within revPBE+vdW differ by less than 0.1 from the corresponding revPBE CNs. The identification of hydration $\mathrm{H}_{2} \mathrm{O}$ molecules was done on the basis of the geometrical cutoff distance of $2.8,3.2,3.7,3.8$, and $3.9 \AA$ between the $\mathrm{O}$ atoms of solvating $\mathrm{H}_{2} \mathrm{O}$ molecules and each cation for $\mathrm{Li}^{+}, \mathrm{Na}^{+}, \mathrm{K}^{+}, \mathrm{Rb}^{+}$, and $\mathrm{Cs}^{+}$, respectively. These distances correspond clearly to the first minima of the RDFs $g_{\mathrm{MO}}(r)$ shown in the upper panel of Fig. 1. We can remark that the decreasing of the intensity of the second peak located at $4.1 \AA$ in $g_{\mathrm{LiO}}(r)$ reduces the over-structuring of $\mathrm{H}_{2} \mathrm{O}$ molecules around $\mathrm{Li}^{+}$observed in a previous work. ${ }^{48}$ On the other hand, while no noticeable changes arise for the distribution of $\mathrm{H}$ atoms around the lighter alkali cations $\mathrm{Li}^{+}$and $\mathrm{Na}^{+}$, the shape of the first peak of $g_{\mathrm{MH}}(r)$ for $\mathrm{M}^{+}=\mathrm{K}^{+}, \mathrm{Rb}^{+}$, and $\mathrm{Cs}^{+}$(each located at 3.4, 3.5, and $3.6 \AA$, respectively) turns out to be modified, particularly for $\mathrm{Cs}^{+}$, if vdWCs are taken into account. This change in the distribution of $\mathrm{H}$ atoms around the cations is accompanied by a change of the tilt angle $\psi$ of hydration $\mathrm{H}_{2} \mathrm{O}$ molecules. Figure 2 shows the distribution of the tilt angle $\psi$ defined as sketched in the inset, namely, $\psi$ is the angle between the dipole vector of a hydrating $\mathrm{H}_{2} \mathrm{O}$ molecule and the vector pointing from the $\mathrm{O}$ atom of $\mathrm{H}_{2} \mathrm{O}$ to the cation. Upon the addition of vdWCs, the distribution of $\psi$ changes in a rather complicated way. For instance, in the case of $\mathrm{Na}^{+}$, water molecules having $\psi \sim 130^{\circ}$ increase in the first hydration shell, while those with $\psi \sim 90^{\circ}$ slightly decrease. Conversely, for $\mathrm{Li}^{+}, \mathrm{K}^{+}$, and $\mathrm{Rb}^{+}$, the two distributions of $\psi$ obtained

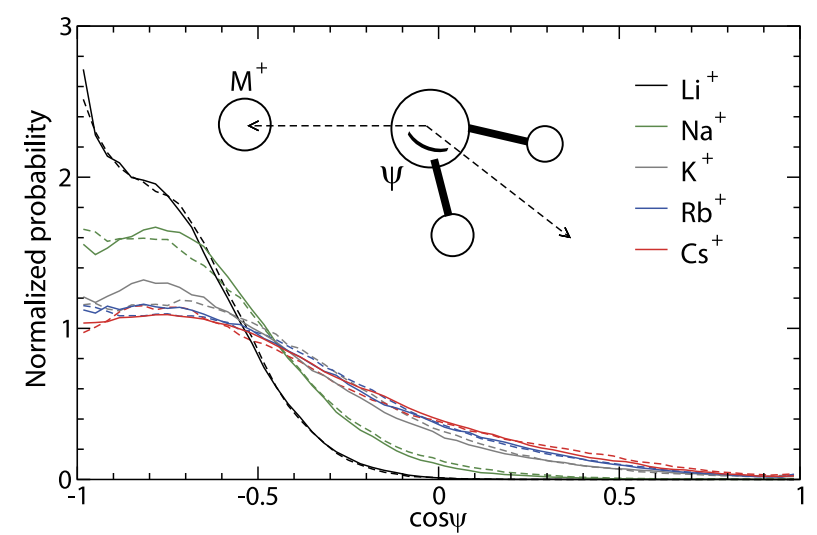

FIG. 2. Distribution of the cosine of the tilt angle $\psi$ between the dipole vector of a hydrating $\mathrm{H}_{2} \mathrm{O}$ molecule and the vector pointing from the $\mathrm{O}$ atom of this water molecule to the cation. Dashed lines refer to revPBE and solid lines to revPBE+vdW. 
within the revPBE and revPBE+vdW schemes are nearly identical except around $\cos \psi=-0.8\left(\psi \sim 143^{\circ}\right)$. Instead, for $\mathrm{Cs}^{+}, \mathrm{H}_{2} \mathrm{O}$ molecules with $80^{\circ} \leq \psi \leq 120^{\circ}$ clearly increase in the first hydration shell when vdWCs are included, indicating that the long-range dispersion forces make $\mathrm{H}_{2} \mathrm{O}$ molecules surrounding $\mathrm{Cs}^{+}$more mobile, in line with the observations reported in the ongoing discussion.

More pronounced differences in the peak intensity of $\mathrm{RDFs}$ due to the use of different $\mathrm{XC}$ functionals were observed in the RDFs of the solvent $g_{\mathrm{OO}}(r)$ and $g_{\mathrm{OH}}(r)$. The first and second peaks of $g_{\mathrm{OO}}(r)$, located, respectively, at 2.8 and $4.4 \AA$ and the second peak of $g_{\mathrm{OH}}(r)$ at $1.8 \AA$ for revPBE and revPBE $+\mathrm{vdW}$ are larger than the corresponding peaks obtained within the GGA after Hamprecht-Cohen-TozerHandy ${ }^{86}$ (HCTH) as previously reported. ${ }^{54,55}$ Indeed, similar $\mathrm{XC}$ functional dependences of the peak intensity (and positions) of RDFs have already been observed for AW. ${ }^{11,87-89}$ A new perspective offered by the present simulations is that by adding vdWCs, the shape of $g_{\mathrm{OO}}(r)$ varies for distances $r \geq 3.0 \AA$ while $g_{\mathrm{OH}}(r)$ is less sensitive to $\mathrm{vdW}$ dispersions. The modification of $g_{\mathrm{OO}}(r)$ induced by our vdWCs is more evident for $\mathrm{Cs}^{+}$as displayed in Fig. 3. The observed reduction of the structure around the second peak of $g_{\mathrm{OO}}(r)$ indicates that the vdW interactions allow $\mathrm{H}_{2} \mathrm{O}$ molecules to fluctuate more freely inside the H-bond network of AW, affecting not only the characteristic tetrahedral coordination but also the dynamical properties as discussed below.

Before focusing on the dynamical properties, it is worthy of note to pay attention to the influence of vdWCs on the

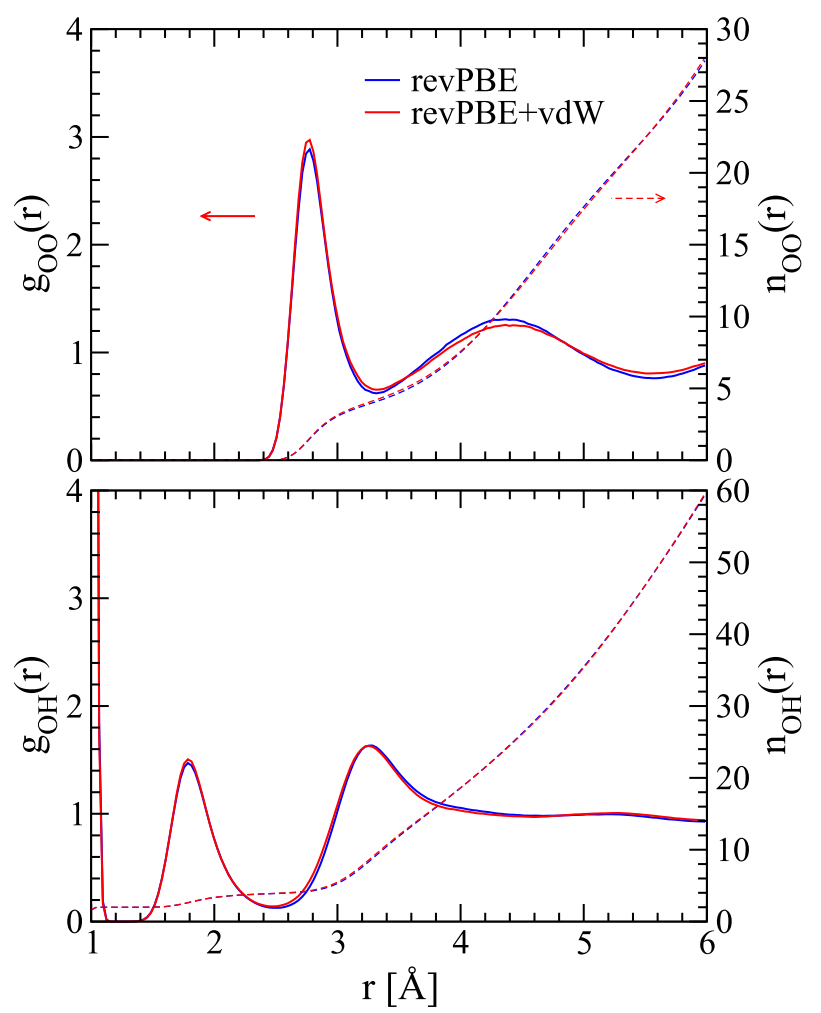

FIG. 3. Computed RDFs $g_{\mathrm{OO}}(r)$ (upper) and $g_{\mathrm{OH}}(r)$ (lower) for our solvated $\mathrm{Cs}^{+}$ions obtained within revPBE (blue) and revPBE+vdW (red). Dashed lines in each panel represent the corresponding CNs $n(r)$ obtained by integrating the RDFs. short-range order of $\mathrm{H}_{2} \mathrm{O}$ molecules. To this aim, a better insight is given by the distribution of the orientational order parameter $q$ defined as ${ }^{90} q=1-\frac{3}{8} \sum_{j=1}^{3} \sum_{k=j+1}^{4}\left(\cos \theta_{j k}+\frac{1}{3}\right)^{2}$, where $\theta_{j k}$ is the $\mathrm{O}-\mathrm{O}-\mathrm{O}$ angle formed by joining an $\mathrm{O}$ atom of a given molecule and those of its nearest neighbors $j$ and $k(\leq 4)$. The average value of $q$ varies between 0 in an ideal gas and 1 in a perfect tetrahedral network; hence, it can be used as a measure of tetrahedrality for local coordination structure. The $f(q)$ distribution presented in Fig. 4 shows two features at $q \simeq 0.85$ and 0.52 . The peak at 0.85 corresponds clearly (from its definition) to an almost perfect tetrahedral coordination, which is also the dominant feature in pure AW. ${ }^{90,91}$ Instead, the shoulder at 0.52 represents a significantly distorted coordination arising from the disruption of the continuous H-bond network due not only to the presence of the cations but also to the effect of $\mathrm{vdW}$ dispersion interactions. This is corroborated by a comparison with former FPMD simulations of pure water, ${ }^{91}$ where a crossover was eventually observed to occur between H-bonded and simple-liquid-like liquids by increasing the temperature. Moreover, the average value of $q$ for each system, indicated as $\langle q\rangle$ in Table I, suggests that the presence of $\mathrm{K}^{+}, \mathrm{Rb}^{+}$, and $\mathrm{Cs}^{+}$in water at ambient conditions reduces more efficiently the tetrahedral order of the coordination shell of $\mathrm{H}_{2} \mathrm{O}$ molecules, in good accord with their prominent structure breaking ability.

Figures 5 and 6 show, respectively, the mean square displacement (MSD) $\left\langle\left(\mathbf{R}_{i}(t)-\mathbf{R}_{i}(0)\right)^{2}\right\rangle$ and the decay of the second orientational correlation function $C_{2}^{\mathrm{HH}}(t)=\left\langle P_{2}\left(\mathbf{e}_{i}(t)\right.\right.$. $\left.\left.\mathbf{e}_{i}(0)\right)\right\rangle$ of $\mathrm{H}_{2} \mathrm{O}$ molecules for our aqueous solutions of alkali ions. Here, $\mathbf{R}_{i}$ and $\mathbf{e}_{i}$ are the coordinates of the center of mass and a unit vector along the $\mathrm{HH}$ vector connecting two $\mathrm{H}$ atoms of the $i$ th $\mathrm{H}_{2} \mathrm{O}$ molecule, respectively. $P_{2}(\cdot)$ is the second Legendre function, and $\langle\cdot\rangle$ means the ensemble average. Consistent with the dependence of water structures on the GGA adopted, $\mathrm{H}_{2} \mathrm{O}$ molecules simulated using either revPBE or revPBE $+\mathrm{vdW}$ exhibit slower diffusional and reorientational motions than those obtained within a HCTH approach. Our estimated self-diffusion coefficient $D$ and reorientational correlation time $\tau_{2 R}$ for $\mathrm{H}_{2} \mathrm{O}$ molecules in our solutions are summarized in Table I. Here, the values of $D$ and $\tau_{2 R}$

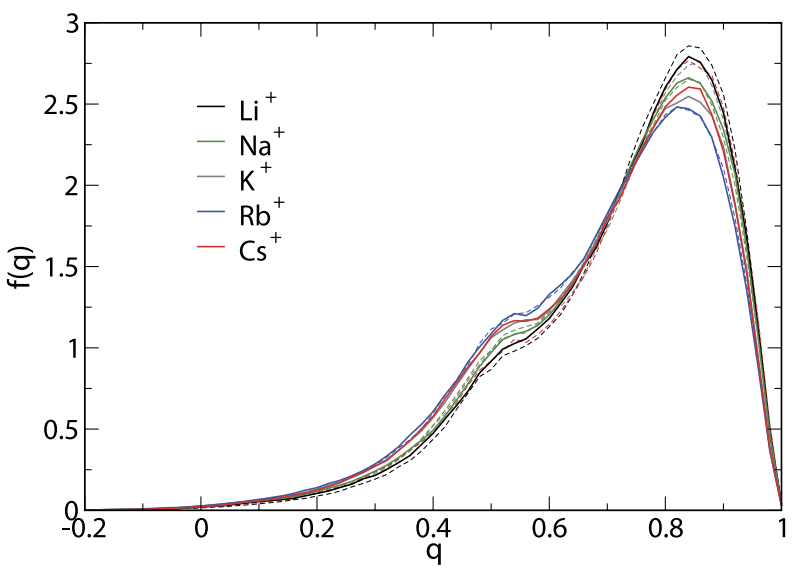

FIG. 4. Distribution of the orientational order parameter $q$ representing the tetrahedrality of the coordination shell of water molecules obtained within revPBE (dashed) and revPBE+vdW (solid). 
TABLE I. Computed self-diffusion coefficient $D$ (in $10^{-5} \mathrm{~cm}^{2} \mathrm{~s}^{-1}$ ), reorientational correlation time $\tau_{2 R}$ (in ps), and average tetrahedrality parameter $\langle q\rangle$ of water molecules in our alkali metal ions solutions. The experimental values of $D$ are taken for aqueous solutions of alkali chlorides at the concentration of $2.0 \mathrm{~m}$. For comparison, the data for pure AW are included.

\begin{tabular}{lccccc}
\hline \hline Ion & Quantity & HCTH & revPBE & revPBE+vdW & Expt. \\
\hline $\mathrm{Li}^{+}$ & $D$ & $1.2^{\mathrm{a}}$ & 0.75 & 0.92 & $2.0^{\mathrm{b}}$ \\
& $\tau_{2 R}$ & $4.2^{\mathrm{a}}$ & 7.7 & 6.6 & $4.5^{\mathrm{c}}$ \\
& $\langle q\rangle$ & 0.68 & 0.73 & 0.72 & $\ldots$ \\
$\mathrm{Na}^{+}$ & $D$ & $1.5^{\mathrm{a}}$ & 0.82 & 0.73 & $2.1^{\mathrm{b}}$ \\
& $\tau_{2 R}$ & $4.6^{\mathrm{a}}$ & 6.9 & 7.3 & $3.4^{\mathrm{c}}$ \\
& $\langle q\rangle$ & 0.65 & 0.71 & 0.71 & $\ldots$ \\
$\mathrm{K}^{+}$ & $D$ & $1.4^{\mathrm{a}}$ & 0.74 & 1.1 & $2.3^{\mathrm{b}}$ \\
& $\tau_{2 R}$ & $4.8^{\mathrm{a}}$ & 7.6 & 5.6 & $1.6^{\mathrm{c}}$ \\
& $\langle q\rangle$ & 0.68 & 0.71 & 0.70 & $\ldots$ \\
$\mathrm{Rb}^{+}$ & $D$ & $1.4^{\mathrm{d}}$ & 1.1 & 1.1 & $\ldots$ \\
& $\tau_{2 R}$ & $3.4^{\mathrm{d}}$ & 5.4 & 5.3 & $1.5^{\mathrm{c}}$ \\
& $\langle q\rangle$ & 0.68 & 0.69 & 0.69 & $\ldots$ \\
$\mathrm{Cs}^{+}$ & $D$ & $1.5^{\mathrm{d}}$ & 0.66 & 1.1 & $2.5^{\mathrm{b}}$ \\
& $\tau_{2 R}$ & $4.9^{\mathrm{d}}$ & 8.3 & 5.7 & $1.6^{\mathrm{c}}$ \\
& $\langle q\rangle$ & 0.68 & 0.72 & 0.70 & $\ldots$ \\
Pure water & $D$ & $1.4^{\mathrm{a}}$ & 0.84 & 0.72 & $2.2^{\mathrm{b}}$ \\
& $\tau_{2 R}$ & $3.5^{\mathrm{a}}$ & 6.6 & 9.1 & $2.0^{\mathrm{c}}$ \\
& $\langle q\rangle$ & 0.70 & 0.73 & 0.74 & $0.58^{\mathrm{e}}$ \\
& & & &
\end{tabular}

a'Ikeda et al. ${ }^{54}$

${ }^{b}$ Valiev and Emel'yanov. ${ }^{92}$

${ }^{\mathrm{c}}$ Shimizu and Taniguchi. ${ }^{93}$

${ }^{\mathrm{d}}$ Ikeda and Boero. ${ }^{55}$

${ }^{\text {e}}$ Soper and Benmore. ${ }^{94}$

are computed from the slope of MSD and the exponential decay rate of $C_{2}^{\mathrm{HH}}(t)$, respectively. For comparison, the two quantities computed on a $64 \mathrm{H}_{2} \mathrm{O}$ system for pure $\mathrm{AW}$ are included. The theoretical values of $D$ for $\mathrm{Li}^{+}$, estimated as $1.2,0.75$, and $0.92 \times 10^{-5} \mathrm{~cm}^{2} \mathrm{~s}^{-1}$ using HCTH, revPBE, and revPBE $+\mathrm{vdW}$ (in this order) correspond to only $60 \%, 38 \%$, and $46 \%$ of the experimental value of $2.0 \times 10^{-5} \mathrm{~cm}^{2} \mathrm{~s}^{-1}$ extracted from spin-echo relaxation measurements for lithium chloride aqueous solution at the concentration of $2.0 \mathrm{~m} .{ }^{92}$ Although the inclusion of vdWCs systematically improves

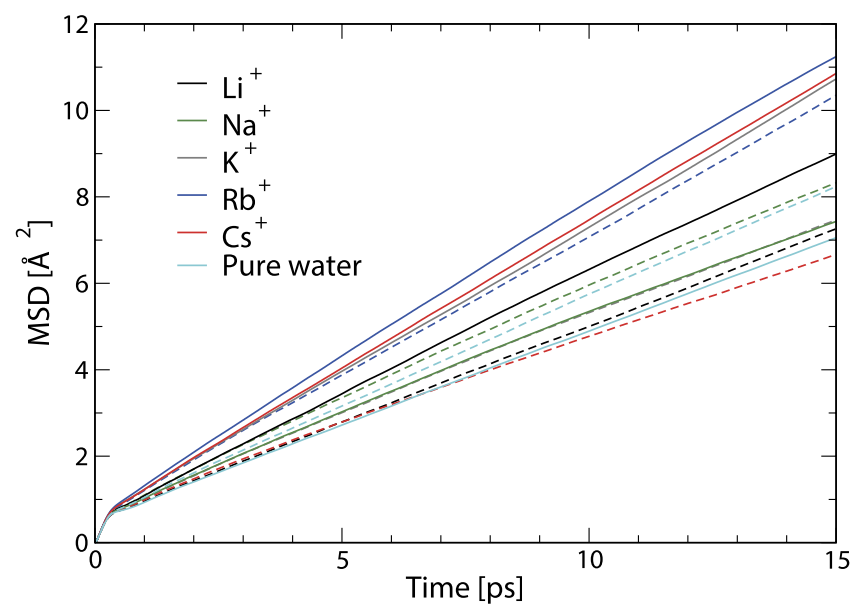

FIG. 5. Computed MSD of $\mathrm{H}_{2} \mathrm{O}$ molecules for alkali metal ions in solution and for pure water using revPBE (dashed) and revPBE+vdW (solid). The line for $\mathrm{K}^{+}$corresponding to revPBE simulations overlaps with that for $\mathrm{Na}^{+}$using revPBE+vdW.

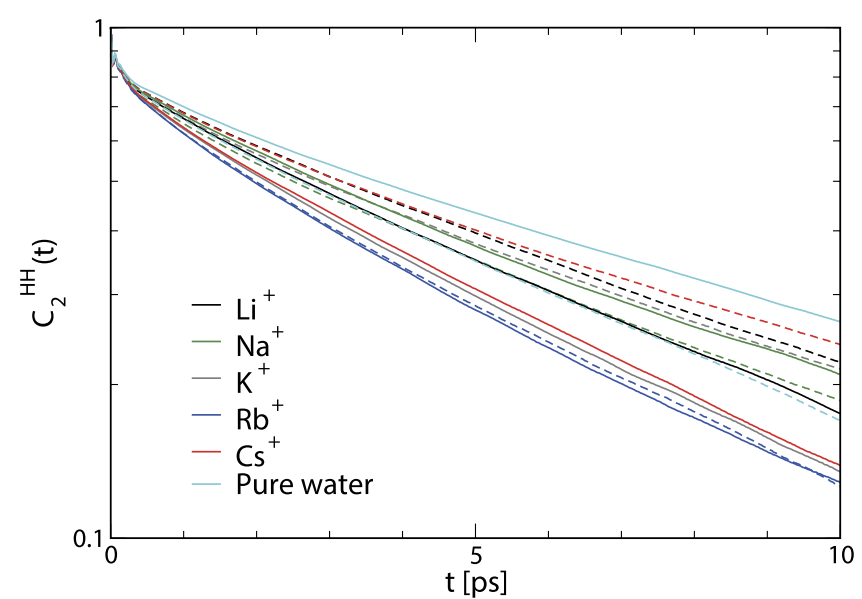

FIG. 6. Computed $C_{2}^{\mathrm{HH}}(t)$ of $\mathrm{H}_{2} \mathrm{O}$ molecules for alkali metal ions in solution and for pure water using the revPBE (dashed) and the revPBE+vdW (solid) schemes.

the same $\mathrm{XC}$ functional in which $\mathrm{vdW}$ interactions are neglected, the underestimation is still clearly present. This is consistent with the theoretical $D$ values of AW estimated from FPMD reported in the literature $\mathrm{e}^{11,31,70,71,87}$ and can be regarded as the standard performance of the present stateof-the-art GGAs. Similarly, for the other alkali cations, our estimated $D$ values amount to only roughly $1 / 2 \sim 1 / 3$ of the experimental ones, measured on aqueous solutions of alkali chlorides (see Table I). We can also add that the systematic underestimates of $D$ might be partially due to finite size effects, ${ }^{89,95}$ choice of the XC functional (see Table I), and the lack of nuclear quantum effects. ${ }^{95}$ Another point worthy of note is that our computed MSDs for revPBE (and also HCTH) shown in Fig. 5 do not change systematically from $\mathrm{Li}^{+}$to $\mathrm{Cs}^{+}$, contrary to experiments. As for $\mathrm{Na}^{+}$, the computed $D$ appears not to be improved by including our vdWCs. However, compared to the corresponding values of revPBE and revPBE+vdW for pure $\mathrm{AW}$, we find that the presence of $\mathrm{Na}^{+}$leads to a minor influence on the diffusive motions of $\mathrm{H}_{2} \mathrm{O}$ molecules, in line with the experimental knowledge that $\mathrm{Na}^{+}$ions are in close proximity of the boundary between structure-maker and -breaker. In contrast, simulations using revPBE+vdW show that the MSD for $\mathrm{K}^{+}, \mathrm{Rb}^{+}$, and $\mathrm{Cs}^{+}$ aqueous solutions increases more rapidly than that for the corresponding solutions of light alkali metals. This is a rather clear indicator of the improvement represented by our vdWCs in the description of diffusive motions of water in aqueous solutions. Note that our revPBE+vdW gives smaller $D$ value (by $0.12 \times 10^{-5} \mathrm{~cm}^{2} \mathrm{~s}^{-1}$ ) for pure AW than the bare revPBE, in agreement with the result ${ }^{31}$ obtained with dispersion corrected atom centered potentials ${ }^{18}$ within the same revPBE functional used here. Interestingly, our preliminary simulations for pure AW using PBE, Becke-Lee-Yang-Parr (BLYP), and HCTH suggest that the dependence of the computed $D$ on the XC functionals tends to be reduced by including our vdWCc. Yet, the XC functional has a major effect that cannot be generally changed by the small corrections represented by the inclusion of vdW dispersion. Hence, we can conclude that weak intermolecular interactions must be included for proper simulations of aqueous systems. 
Improvements due to the inclusion of vdWCs are evident also in the reorientational motions of $\mathrm{H}_{2} \mathrm{O}$ molecules, shown in Fig. 6. Indeed, the $C_{2}^{\mathrm{HH}}(t)$ computed using revPBE+vdW decays more rapidly for heavy alkali metal ions, indicating that $\tau_{2 R}$ tends to decrease when the atomic number of alkali metal ions increases, in good agreement with the observed trend. Nonetheless, our $\tau_{2 R}$ estimated as 6.6 for $\mathrm{Li}^{+}, 7.3$ for $\mathrm{Na}^{+}, 5.6$ for $\mathrm{K}^{+}, 5.3$ for $\mathrm{Rb}^{+}$, and $5.7 \mathrm{ps}$ for $\mathrm{Cs}^{+}$is overestimated by a factor of $2 \sim 3$ with respect to the experimental correlation time extracted by NMR spectroscopy on alkali bromide dilute aqueous solutions. ${ }^{93}$ Summarizing, our estimations of $D$ and $\tau_{2 R}$ clearly show that our computational approach of revPBE+vdW improves systematically the performance of the vdW-uncorrected functional. Yet, this improvement is quantitatively not fully satisfactory. This arises partly from the still too strong tendency to form the tetrahedrally coordinated water in our aqueous solutions; such an issue is confirmed by a careful reanalysis of the trajectories obtained in our previous studies ${ }^{54,55}$ making use of the HCTH functional. In fact, HCTH gives $\langle q\rangle$ values $(\simeq 0.68)$ generally smaller than revPBE+vdW (see Table I), underscoring the importance in the choice of the XC functional on which, on a second instance, vdWCs can be added. Nonetheless, our revPBE+vdW represents a practical and cheap alternative to more elaborated hybrid XC functionals resulting in $\langle q\rangle$ and $D$ close to ours ${ }^{34}$ (but computationally much more demanding), thus providing a viable FP-based way of including vdWCs beyond simple (semi)empirical ones.

\section{CONCLUSIONS}

We presented an accurate analysis of (simulated) aqueous solutions of alkali metal ions aimed to critically assess the performance of our FP-based vdWCs implemented in the CPMD code. These studies allow to rationalize the role of long range dispersion forces in the solute-solvent interactions. The influence of vdWCs on the structural and dynamical properties differs significantly depending on the structuremaking and -breaking ability of the various alkali metal ions. For $\mathrm{Li}^{+}$, which is considered as a strong structure maker, the non-negligible influence of long-range dispersion interactions is pretty evident in the dynamics of $\mathrm{H}_{2} \mathrm{O}$ molecules and in their clustering. In contrast, for $\mathrm{Na}^{+}$, which is close to the boundary between structure maker and breaker, vdWCs play a minor role on both the structural and dynamical properties. On the other hand, for alkali ions heavier than $\mathrm{K}^{+}$, representative of structure breakers, the over-structured second solvation shell of water is reduced to some extent, consistent with the enhanced diffusion and reorientation of $\mathrm{H}_{2} \mathrm{O}$ molecules, upon the inclusion of vdWCs, in line with experiments. Note that besides heavy alkali ions, many anions are regarded as structure breakers. Therefore, the description of their hydration behavior is also expected to be substantially improved by including our vdWCs.

Although the experimentally observed trend of the $D$ and $\tau_{2 R}$ of water in aqueous solutions of alkali metal ions is reasonably reproduced by our approach, the performance of revPBE+vdW is quantitatively not yet satisfactory. The computed $D$ values of water are typically underestimated by as much as $1 / 2 \sim 1 / 3$ of experiment ones and the values of $\tau_{2 R}$ are overestimated by a factor of $2 \sim 3$. Nevertheless, the importance of the vdWCs scheme presented here allows for a non-negligible improvement of the bare GGAs for solutions, interfaces, and layered compounds. ${ }^{96}$ Moreover, our vdWCs approach is generally applicable to a wide variety of systems at a nearly negligible computational cost with respect to the computational workload of the corresponding bare GGAs and also in comparison with other FP vdW schemes.

\section{ACKNOWLEDGMENTS}

The calculations reported in this paper were performed at the JAEA supercomputer facility. M.B. is grateful to GENCI (Grand Equipement National de Calcul Intensif) under Grant No. x2015096092.

${ }^{1}$ R. W. Gurney, Ionic Processes in Solution (McGraw-Hill, New York, 1953).

${ }^{2}$ J. Burgess, Metal Ions in Solution (Ellis Horwood, New York, 1978).

${ }^{3}$ B. E. Conway, Ionic Hydration in Chemistry and Biophysics, Studies in Physical and Theoretical Chemistry (Elsevier, Amsterdam, 1981), Vol. 12.

${ }^{4}$ Y. Marcus, Ion Solvation (Wiley, Chichester, 1986).

${ }^{5}$ Y. Marcus, Chem. Rev. 88, 1475 (1988).

${ }^{6}$ D. T. Richens, The Chemistry of Aqua Ions (Wiley, Chichester, 1997).

${ }^{7}$ H. Ohtaki and T. Radnai, Chem. Rev. 93, 1157 (1993).

${ }^{8}$ M. Boero, T. Ikeda, E. Ito, and K. Terakura, J. Am. Chem. Soc. 128, 16798 (2006).

${ }^{9}$ Y. Marcus, Chem. Rev. 109, 1346 (2009).

${ }^{10}$ B. A. F. Previdello, E. G. Machado, and H. Varela, RSC Adv. 4, 15271 (2014).

${ }^{11}$ M. Sprik, J. Hutter, and M. Parrinello, J. Chem. Phys. 105, 1142 (1996).

${ }^{12}$ P. L. Silvestrelli and M. Parrinello, Phys. Rev. Lett. 82, 3308 (1999).

${ }^{13}$ P. L. Silvestrelli and M. Parrinello, J. Chem. Phys. 111, 3572 (1999).

${ }^{14}$ D. Prendergast, J. C. Grossman, and G. Galli, J. Chem. Phys. 123, 014501 (2005).

${ }^{15}$ H.-S. Lee and M. E. Tuckerman, J. Chem. Phys. 126, 164501 (2007).

${ }^{16}$ W. Kohn and L. J. Sham, Phys. Rev. 140, A1133 (1965).

${ }^{17}$ S. Grimme, J. Comput. Chem. 25, 1463 (2004).

${ }^{18}$ O. A. von Lilienfeld, I. Tavernelli, U. Rothlisberger, and D. Sebastiani, Phys. Rev. Lett. 93, 153004 (2004).

${ }^{19}$ R. W. Williams and D. Malhotra, Chem. Phys. 327, 54 (2006).

${ }^{20}$ P. L. Silvestrelli, J. Phys. Chem. A 113, 5224 (2009).

${ }^{21}$ S. Grimme, J. Antony, S. Ehrlich, and H. Krieg, J. Chem. Phys. 132, 154104 (2010).

${ }^{22}$ A. Ambrosetti and P. L. Silvestrelli, Phys. Rev. B 85, 073101 (2012).

${ }^{23}$ M. Dion, H. Rydberg, E. Schroder, D. C. Langreth, and B. I. Lundqvist, Phys. Rev. Lett. 92, 246401 (2004).

${ }^{24}$ O. A. Vydrov and T. Van Voorhis, Phys. Rev. Lett. 103, 063004 (2009).

${ }^{25}$ A. Tkatchenko, R. A. DiStasio, R. Car, and M. Scheffler, Phys. Rev. Lett. 108, 236402 (2012).

${ }^{26}$ I. Hamada, Phys. Rev. B 89, 121103 (2014).

${ }^{27}$ J. Schmidt, J. VandeVondele, I. F. W. Kuo, D. Sebastiani, J. I. Siepmann, J. Hutter, and C. J. Mundy, J. Phys. Chem. B 113, 11959 (2009).

${ }^{28}$ J. Wang, G. Roman-Perez, J. M. Soler, E. Artacho, and M.-V. FernandezSerra, J. Chem. Phys. 134, 024516 (2011).

${ }^{29}$ S. Yoo and S. S. Xantheas, J. Chem. Phys. 134, 121105 (2011).

${ }^{30}$ C. Zhang, J. Wu, G. Galli, and F. Gygi, J. Chem. Theory Comput. 7, 3054 (2011).

${ }^{31}$ I. C. Lin, A. P. Seitsonen, I. Tavernelli, and U. Rothlisberger, J. Chem. Theory Comput. 8, 3902 (2012).

${ }^{32}$ Z. Ma, Y. Zhang, and M. E. Tuckerman, J. Chem. Phys. 137, 044506 (2012).

${ }^{33}$ K. Forster-Tonigold and A. Gros, J. Chem. Phys. 141, 064501 (2014).

${ }^{34}$ R. A. DiStasio, B. Santra, Z. Li, X. Wu, and R. Car, J. Chem. Phys. 141, 084502 (2014).

${ }^{35}$ G. Miceli, S. de Gironcoli, and A. Pasquarello, J. Chem. Phys. 142, 034501 (2015).

${ }^{36}$ E. Guardia, I. Skarmoutsos, and M. Masia, J. Phys. Chem. B 119, 8926 (2015).

${ }^{37}$ A. Bankura, A. Karmakar, V. Carnevale, A. Chandra, and M. L. Klein, J. Phys. Chem. C 118, 29401 (2014). 
${ }^{38}$ A. P. Gaiduk, F. Gygi, and G. Galli, J. Phys. Chem. Lett. 6, 2902 (2015).

${ }^{39}$ W. Stumm and J. J. Morgan, Aquatic Chemistry, 3rd ed. (Wiley, New York, 1996).

${ }^{40}$ J. Mulet, V. Llopis-Torregrosa, C. Primo, M. Marques, and L. Yenush, Curr. Genet. 59, 207 (2013).

${ }^{41}$ J. Tymoczko, V. Colic, A. Ganassin, W. Schuhmann, and A. S. Bandarenka, Catal. Today 244, 96 (2015).

${ }^{42}$ K. Moriwaki, M. Nomoto, S. Ishizuka, A. Mukai, K. Sato, H. Kobayashi, and S. Niki, Sol. Energy Mater. Sol. Cells 133, 21 (2015).

${ }^{43}$ A. W. Omta, M. F. Kropman, S. Woutersen, and H. J. Bakker, J. Chem. Phys. 119, 12457 (2003).

${ }^{44}$ A. W. Omta, M. F. Kropman, S. Woutersen, and H. J. Bakker, Science 301, 347 (2003).

${ }^{45}$ I. Waluyo, D. Nordlund, U. Bergmann, D. Schlesinger, L. G. M. Pettersson, and A. Nilsson, J. Chem. Phys. 140, 244506 (2014).

${ }^{46}$ L. M. Ramaniah, M. Bernasconi, and M. Parrinello, J. Chem. Phys. 111, 1587 (1999).

${ }^{47}$ J. A. White, E. Schwegler, G. Galli, and F. Gygi, J. Chem. Phys. 113, 4668 (2000).

${ }^{48}$ A. P. Lyubartsev, K. Laasonen, and A. Laaksonen, J. Chem. Phys. 114, 3120 (2001).

${ }^{49}$ R. Vuilleumier and M. Sprik, J. Chem. Phys. 115, 3454 (2001).

${ }^{50}$ Y. Liu, H. Lu, Y. Wu, T. Hu, and Q. Li, J. Chem. Phys. 132, 124503 (2010).

${ }^{51}$ Y. Zeng, J. Hu, Y. Yuan, X. Zhang, and S. Ju, Chem. Phys. Lett. 538, 60 (2012).

${ }^{52}$ A. Bankura, V. Carnevale, and M. L. Klein, J. Chem. Phys. 138, 014501 (2013).

${ }^{53}$ C. Allolio, N. Salas-Illanes, Y. S. Desmukh, M. R. Hansen, and D. Sebastiani, J. Phys. Chem. B 117, 9939 (2013).

${ }^{54}$ T. Ikeda, M. Boero, and K. Terakura, J. Chem. Phys. 126, 034501 (2007).

${ }^{55}$ T. Ikeda and M. Boero, J. Chem. Phys. 137, 041101 (2012).

${ }^{56}$ R. Scipioni, D. A. Schmidt, and M. Boero, J. Chem. Phys. 130, 024502 (2009).

${ }^{57}$ D. A. Schmidt, R. Scipioni, and M. Boero, J. Phys. Chem. A 113, 7725 (2009).

${ }^{58}$ R. Car and M. Parrinello, Phys. Rev. Lett. 55, 2471 (1985).

${ }^{59}$ CPMD; IBM Corporation, 1990-2014; and MPI für Festkörperforshung Stuttgart, 1997-2001. http://www.cpmd.org.

${ }^{60}$ J. P. Perdew, K. Burke, and M. Ernzerhof, Phys. Rev. Lett. 77, 3865 (1996).

${ }^{61}$ Y. Zhang and W. Yang, Phys. Rev. Lett. 80, 890 (1998).

${ }^{62}$ N. Marzari and D. Vanderbilt, Phys. Rev. B 56, 12847 (1997).

${ }^{63}$ R. Resta and S. Sorella, Phys. Rev. Lett. 82, 370 (1999).

${ }^{64}$ N. Marzari, A. A. Mostofi, J. R. Yates, I. Souza, and D. Vanderbilt, Rev. Mod. Phys. 84, 1419 (2012).
${ }^{65}$ P. L. Silvestrelli, Phys. Rev. Lett. 100, 053002 (2008).

${ }^{66}$ N. Troullier and J. L. Martins, Phys. Rev. B 43, 1993 (1991).

${ }^{67}$ S. Goedecker, M. Teter, and J. Hutter, Phys. Rev. B 54, 1703 (1996).

${ }^{68}$ C. Hartwigsen, S. Goedecker, and J. Hutter, Phys. Rev. B 58, 3641 (1998).

${ }^{69}$ F. A. Bornemann and C. Schutte, Numer. Math. 78, 359 (1998).

${ }^{70}$ J. C. Grossman, E. Schwegler, E. W. Draeger, F. Gygi, and G. Galli, J. Chem. Phys. 120, 300 (2004).

${ }^{71}$ E. Schwegler, J. C. Grossman, F. Gygi, and G. Galli, J. Chem. Phys. 121, 5400 (2004).

${ }^{72}$ G. Makov and M. C. Payne, Phys. Rev. B 51, 4014 (1995).

${ }^{73}$ S. Nosé, Mol. Phys. 52, 255 (1984).

${ }^{74}$ S. Nosé, J. Chem. Phys. 81, 511 (1984).

${ }^{75}$ W. G. Hoover, Phys. Rev. A 31, 1695 (1985).

${ }^{76}$ G. J. Martyna, M. L. Klein, and M. Tuckerman, J. Chem. Phys. 97, 2635 (1992).

${ }^{77}$ A. H. Narten, F. Vaslow, and H. A. Levy, J. Chem. Phys. 58, 5017 (1973).

${ }^{78}$ Y. Kameda and O. Uemura, Bull. Chem. Soc. Jpn. 66, 384 (1993).

${ }^{79}$ G. Pálinkás, T. Radnai, and H. Hajdu, Z. Naturforsch., A 35, 107 (1980).

${ }^{80}$ Y. Kameda, K. Sugawara, T. Usuki, and O. Uemura, Bull. Chem. Soc. Jpn. 71, 2769 (1998).

${ }^{81}$ N. T. Skipper and G. W. Neilson, J. Phys.: Condens. Matter 1, 4141 (1989).

${ }^{82}$ N. Ohtomo and K. Arakawa, Bull. Chem. Soc. Jpn. 53, 1789 (1980).

${ }^{83}$ G. Paschina, G. Piccaluga, G. Pinna, and M. Magini, Chem. Phys. Lett. 98, 157 (1983).

${ }^{84}$ G. W. Neilson and N. Skipper, Chem. Phys. Lett. 114, 35 (1985).

${ }^{85}$ N. Ohtomo and K. Arakawa, Bull. Chem. Soc. Jpn. 52, 2755 (1979).

${ }^{86}$ F. A. Hamprecht, A. J. Cohen, D. J. Tozer, and N. C. Handy, J. Chem. Phys. 109, 6264 (1998).

${ }^{87}$ A. D. Boese, N. L. Doltsinis, N. C. Handy, and M. Sprik, J. Chem. Phys. 112, 1670 (2000).

${ }^{88}$ J. VandeVondele, F. Mohamed, M. Krack, J. Hutter, M. Sprik, and M. Parrinello, J. Chem. Phys. 122, 014515 (2005).

${ }^{89}$ T. D. Kühne, M. Krack, and M. Parrinello, J. Chem. Theory Comput. 5, 235 (2009).

${ }^{90}$ J. R. Errington and P. G. Debenedetti, Nature 409, 318 (2001).

${ }^{91}$ T. Ikeda, Y. Katayama, H. Saitoh, and K. Aoki, J. Chem. Phys. 132, 121102 (2010).

${ }^{92}$ K. A. Valiev and M. I. Emel'yanov, J. Struct. Chem. 5, 625 (1965).

${ }^{93}$ A. Shimizu and Y. Taniguchi, Bull. Chem. Soc. Jpn. 64, 221 (1991).

${ }^{94}$ A. K. Soper and C. J. Benmore, Phys. Rev. Lett. 101, 065502 (2008).

${ }^{95}$ M. Del Ben, J. Hutter, and J. VandeVondele, J. Chem. Phys. 143, 054506 (2015).

${ }^{96}$ T. Ikeda, S. Suzuki, and T. Yaita, J. Phys. Chem. A 119, 8369 (2015). 